\title{
Large-scale structure, the cosmic microwave background and primordial non-Gaussianity
}

\author{
Licia Verde, ${ }^{1}$ Limin Wang, ${ }^{2}$ Alan F. Heavens ${ }^{1}$ and Marc Kamionkowski ${ }^{2}$ \\ ${ }^{1}$ Institute for Astronomy, University of Edinburgh, Royal Observatory, Blackford Hill, Edinburgh EH9 $3 H J$ \\ ${ }^{2}$ Department of Physics, Columbia University, 538 West 120th Street, New York, NY 10027, USA
}

Accepted 1999 October 25. Received 1999 September 27; in original form 1999 June 3

\begin{abstract}
A B S T R A C T
Cosmic microwave background and large-scale structure data will shortly improve dramatically with the Microwave Anisotropy Probe and Planck Surveyor, and the AngloAustralian 2-Degree Field and Sloan Digital Sky Survey. It is therefore timely to ask which of the microwave background and large-scale structure will provide a better probe of primordial non-Gaussianity. In this paper we consider this question, using the bispectrum as a discriminating statistic. We consider several non-Gaussian models and find that in each case the microwave background will provide a better probe of primordial non-Gaussianity. Our results suggest that if microwave background maps appear Gaussian, then apparent deviations from Gaussian initial conditions in galaxy surveys can be attributed with confidence to the effects of biasing. We demonstrate this precisely for the spatial bispectrum induced by local non-linear biasing.
\end{abstract}

Key words: methods: analytical - cosmic microwave background - cosmology: theory large-scale structure of Universe.

\section{INTRODUCTION}

It is widely accepted that the large-scale structures we observe in the Universe today originated from gravitational evolution of small primordial fluctuations in the matter density. Information about the physical processes that generated these primordial fluctuations can be gleaned by testing whether their statistical distribution is well approximated by a Gaussian random field. In particular, the simplest versions of inflation predict Gaussian initial fluctuations (e.g. Guth \& Pi 1982; Hawking 1982; Starobinsky 1982; Bardeen, Steinhardt \& Turner 1983), but there are other models of inflation (Allen, Grinstein \& Wise 1987; Kofman \& Pogosyan 1988; Salopek, Bond \& Bardeen 1989), and models where structure is seeded by topological defects (Vilenkin 1985; Vachaspati 1986; Hill, Schramm \& Fry 1989; Turok 1989; Albrecht \& Stebbins 1992) that generate non-Gaussian fluctuations. By looking at cosmic microwave background (CMB) anisotropies we can probe cosmic fluctuations at a time when their statistical distribution should have been close to its original form. At present, the limited signal-to-noise ratio or sky coverage of existing experiments is not sufficient to provide conclusive evidence either for or against non-Gaussianity (e.g. Heavens 1998; Ferreira, Magueijo \& Gorski 1998; Kamionkowski \& Jaffe 1998; Pando, Valls-Gabaud \& Fang 1998; Bromley \& Tegmark 1999). An alternative approach is to analyse the present-day statistics of density or velocity fields of large-scale structure (LSS). In principle, this is a more complicated approach, since gravitational instability and bias can introduce non-Gaussian features in an initially Gaussian field, and these may mask the signal we desire to measure. The CMB and LSS data will shortly improve dramatically with the Microwave Anisotropy Probe $(M A P)$ and Planck Surveyor satellites and the Anglo-Australian 2-Degree Field (2dF) and Sloan Digital Sky Survey (SDSS). For this reason it is timely to ask whether the CMB or LSS is the better placed to detect a primordial non-Gaussian signal.

In this paper, we use the skewness and bispectrum to determine whether the CMB or LSS provides a better probe of nonGaussianity. To do so, we consider several models with a primordial non-Gaussianity whose amplitude can be dialed from zero (the Gaussian limit). Throughout we neglect complications of pixel noise and foreground in the CMB, although we include shot noise in LSS. We then calculate the smallest non-Gaussian amplitude that can be detected with a MAP/Planck map and with $2 \mathrm{dF} / \mathrm{SDSS}$. In each case, we find that the smallest non-Gaussian amplitude detectable with the CMB map is smaller than that from a galaxy survey, even if we neglect the complicating effects of biasing.

Of course, there are an infinitude of possible deviations from Gaussianity and we cannot address them all. However, physical mechanisms that produce non-Gaussianity generically produce a non-vanishing bispectrum. Of the correlation functions that are zero for Gaussian fields, the bispectrum is the lowest order and usually the most easily detectable. Although this argument is not fully general, we show that it holds in several non-Gaussian models that have been considered in the recent literature. Our results suggest that if the $\mathrm{CMB}$ maps provided by $M A P$ and 
Planck are consistent with Gaussian initial conditions, then any signatures of non-Gaussianity found in galaxy surveys (apart from those from non-linear clustering) can be attributed to biasing. Even though the overall amplitude of the signal is smaller for the CMB than for LSS, every non-zero detection of CMB bispectrum is a direct detection of primordial non-Gaussianity, while the perturbative non-Gaussian contributions to the LSS bispectrum swamp the desired signal.

\section{THE SKEWNESS}

In order to determine whether a field is Gaussian, we need a discriminating statistic. We shall principally be concerned with the bispectrum, as it is the Fourier counterpart of the lowest-order connected correlation function that generically arises in physical mechanisms that produce non-Gaussianity, and it is able in principle to distinguish between various sources of non-Gaussianity (e.g. primordial, non-linear growth, bias). However, we begin by discussing the skewness in LSS and the CMB. The skewness is related to the bispectrum but contains less information; however, as a real space statistic, it is conceptually simpler. Section 2 is largely for illustration; detailed calculations are presented for the bispectrum in Section 3.

\subsection{Skewness in large-scale structure}

The statistical properties of the fluctuations in the cosmological mass density field $\delta(\boldsymbol{x})=[\rho(\boldsymbol{x})-\bar{\rho}] / \bar{\rho}$ can be characterized by the $n$-point moments, $\left\langle\delta^{n}\right\rangle$. By definition, $\langle\delta(x)\rangle=0$. If the fluctuation field is Gaussian, then the probability distribution for $\delta$ is

$p(\delta)=(\sqrt{2 \pi} \sigma)^{-1} \exp \left[-\delta^{2} /\left(2 \sigma^{2}\right)\right]$,

from which the moments $(n=0,1, \ldots)$ can be calculated to be

$\left\langle\delta^{2 n}\right\rangle=(2 n-1) ! !\left\langle\delta^{2}\right\rangle^{n}=(2 n-1) ! ! \sigma^{2 n}$,

where $\sigma^{2} \equiv\left\langle\delta^{2}\right\rangle$. The odd moments are of course zero. To linear order in perturbation theory, $\delta$ grows by an overall normalization factor, $\delta(\boldsymbol{x}, t)=D(t) \delta\left(\boldsymbol{x}, t_{0}\right)$, so an initially Gaussian distribution will remain Gaussian as long as linear perturbation theory holds.

To higher order in perturbation theory, gravitational instability will induce departures from Gaussianity. To describe the evolution of non-linear fluctuations in perturbation theory we expand the fluctuation field in a series,

$\delta=\delta^{(1)}+\delta^{(2)}+\cdots$,

where $\delta^{(n)} \sim O\left(\delta^{n}\right)$ (e.g. Goroff et al. 1986; Fry \& Scherrer 1994). In the weakly non-linear regime, the series can be truncated to second order. The lowest-order deviation from Gaussianity is described by $\left\langle\delta^{3}\right\rangle$. In the weakly non-linear regime, Gaussian initial conditions give rise to a non-vanishing skewness (e.g. Peebles 1980),

$S_{3} \equiv \frac{\left\langle\delta^{3}\right\rangle}{\left\langle\delta^{2}\right\rangle^{2}}=\frac{34}{7}$

in second-order perturbation theory.

For generic non-Gaussian initial conditions, Fry \& Scherrer (1994) found the skewness in second-order perturbation theory to be

$S_{3}=S_{3,0}+\frac{34}{7}-\frac{26}{21} \frac{\left\langle\delta_{0}^{3}\right\rangle^{2}}{\left\langle\delta_{0}^{2}\right\rangle^{3}}-\frac{8}{7}\left\langle\delta_{0}^{3}\right\rangle \frac{I\left[\xi_{(3) 0}\right]}{\left\langle\delta_{0}^{2}\right\rangle^{3}}+\frac{10}{7} \frac{\xi_{(4) 0}}{\left\langle\delta_{0}^{2}\right\rangle^{2}}+\frac{6}{7} \frac{I\left[\xi_{(4) 0}\right]}{\left\langle\delta_{0}^{2}\right\rangle^{2}}$, where the subscript 0 denotes the quantity linearly evolved from the initial density field [e.g. in an Einstein-de Sitter universe, $\left.\delta_{0}=\delta\left(z_{i}\right)\left(1+z_{i}\right)\right]$ to the present epoch. The quantity $I\left[\xi_{(n) 0}\right]$ $(n=3,4)$ is an integral that depends on the specific linearly evolved connected (or irreducible) three- and four-point functions, $\xi_{(3) 0}$ and $\xi_{(4) 0}$, respectively. (For Gaussian initial conditions, $\xi_{(n) 0}=0$ for $n \geq 3$.) Fry \& Scherrer (1994) find that $I\left[\xi_{(n) 0}\right] \leq$ $\xi_{(n) 0}(0)$ for $n=3,4$ for several non-Gaussian models they explore (see also Gaztanaga \& Fosalba 1998). All terms on the right-hand side of equation (5) are time-independent apart from $S_{3,0}$ which scales like $S_{3,0}(z) \propto S_{3,0}(z=0)(1+z)$ in an Einstein-de Sitter universe.

It is therefore useful to define the time-independent quantities $p_{3}=\xi_{(3), 0} / \sigma^{3}$ (normalized skewness) and $p_{4}=\xi_{(4), 0} / \sigma^{4}$ (normalized kurtosis). When written in terms of the relevant quantities at decoupling (i.e., at $z \simeq 1100$ ),

$S_{3} \simeq \frac{34}{7}+\frac{p_{3}}{1100 \sigma(z=1100)}-d_{1} p_{3}^{2}+d_{2} p_{4}$,

where $d_{1} \simeq d_{2} \simeq 2$.

Now suppose we have a survey of $N$ independent volumes among which the rms fractional density contrast is $\sigma$. Then, from equations (2) and (4), it follows that, in the mildly non-linear regime, the standard error due to cosmic variance with which the skewness can be recovered is

$\Delta S_{3}=\frac{1}{\sqrt{N}} \sqrt{\frac{15}{\sigma^{2}}+17 S_{3}^{2}}$,

where we have used $\Delta\left\langle\delta^{3}\right\rangle=\sqrt{15 \sigma^{6}+10\left\langle\delta^{3}\right\rangle^{2}}$ and $\Delta\left\langle\delta^{2}\right\rangle \sim$ $\sqrt{2 \sigma^{4}}$. Of course, since the mass will be traced by discrete objects (i.e. galaxies), the shot noise will increase the error estimate in equation (7).

So now let us consider the $2 \mathrm{dF}$ and/or SDSS. The present-day skewness in volumes of side $10 h^{-1} \mathrm{Mpc}$ (where $h$ is the Hubble parameter in units of $100 \mathrm{~km} \mathrm{~s}^{-1} \mathrm{Mpc}^{-1}$ ) could be measured with a standard error of at least $\Delta S_{3} \sim 20 N^{-1 / 2} \sim 10^{-1}$ (see also Colombi, Szapudi \& Szalay 1998), where $N$ is the number of such cubes in the survey volume. Thus, equation (6) tells us that a primordial normalized skewness on the $10 \mathrm{~h}^{-1} \mathrm{Mpc}$ scale could be identified in a statistically significant manner in $2 \mathrm{dF}$ and/or SDSS only if $p_{3}$ exceeded about $10^{-2}$.

\subsection{Skewness in the CMB}

For simplicity, consider an Einstein-de Sitter universe. Then a region of comoving size $10 \mathrm{~h}^{-1} \mathrm{Mpc}$ subtends an angle of $0.1^{\circ}$. Now suppose that a full-sky cosmic-variance-limited $0.1^{\circ}$-resolution CMB map (close to the specifications of Planck Surveyor) finds that the distribution of temperature fluctuations $\Delta T / T$ is consistent with Gaussian with a variance $\sigma^{2}=\left\langle(\delta \rho / \rho)^{2} \sim 0.1(\delta T / T)^{2} \sim\right.$ $\left(10^{-4}\right)^{2}$ (where $\delta \rho / \rho$ is the fractional density perturbation at a redshift $z \simeq 1100$, when the $\mathrm{CMB}$ decouples). The largest primordial normalized skewness $p_{3} \equiv\left\langle(\delta \rho / \rho)^{3} / \sigma^{3}\right.$ that would be consistent with such a map is $\sqrt{15 / N_{\text {pix }}} \sim 10^{-3}$, where $N_{\text {pix }} \sim$ $10^{7}$ is the number of $0.1^{\circ} \times 0.1^{\circ}$ pixels. We have neglected instrumental noise and assumed that systematic effects will be under control. Still, a sensitivity to a value as small as $p_{3} \sim 10^{-3}$, or perhaps an order of magnitude larger, is a realistic expectation of CMB maps.

If we compare this with the nominal smallest normalized skewness $p_{3} \sim 10^{-2}$ accessible with LSS, it appears that the CMB 
has perhaps an extra order of magnitude in sensitivity. On the other hand, there may be systematic effects in both the CMB and LSS measurements that may affect both estimates, and our argument was only qualitative. Thus, we conclude from this exercise that the CMB and LSS should provide roughly comparable sensitivity to a primordial skewness on $10 h^{-1} \mathrm{Mpc}$ scales, with perhaps a slight edge to the CMB. In practice, noise and foreground contamination may restrict the $\mathrm{CMB}$ analysis to scales larger than $0.1^{\circ}$, later we will investigate CMB fluctuations on scales larger than $1^{\circ}$. On these physical scales the cosmic variance in LSS would lose all sensitivity to a primordial bispectrum. Since these heuristic arguments are inconclusive to orders of magnitude as to which of the CMB and LSS provides a better probe of primordial non-Gaussianity, we now proceed to consider realistic models more carefully.

\section{CMB AND LSS BISPECTRA}

The skewness is a specific case of the three-point function, and, as such, contains less information. We might therefore hope to learn more by studying the full three-point function. In practice, we prefer to investigate its Fourier-space counterpart, the bispectrum, for reasons that have been rehearsed before (e.g. Matarrese, Verde \& Heavens 1997, hereafter MVH97). In addition, various theoretical models for structure formation yield Fourier-space quantities directly, so the bispectrum allows a more straightforward relation between measurable quantities and theoretical predictions.

\subsection{LSS bispectrum}

We define the Fourier transform of the fractional overdensity perturbation by $\delta_{\boldsymbol{k}}=\int \mathrm{d}^{3} \boldsymbol{x} \delta(\boldsymbol{x}) \exp (-\mathrm{i} \boldsymbol{k} \cdot \boldsymbol{x}) /(2 \pi)^{3}$. The spatial bispectrum $B\left(\boldsymbol{k}_{1}, \boldsymbol{k}_{2}, \boldsymbol{k}_{3}\right)$ is defined by

$\left\langle\delta_{\boldsymbol{k}_{1}} \delta_{\boldsymbol{k}_{2}} \delta_{\boldsymbol{k}_{3}}\right\rangle=B\left(\boldsymbol{k}_{1}, \boldsymbol{k}_{2}, \boldsymbol{k}_{3}\right) \delta^{\mathrm{D}}\left(\boldsymbol{k}_{1}+\boldsymbol{k}_{2}+\boldsymbol{k}_{3}\right)$,

where the angle brackets denotes an ensemble average or, under the fair sample hypothesis, the average over a large volume, and $\delta^{\mathrm{D}}$ is the Dirac delta function.

To second order in perturbation theory, the bispectrum may be written as the sum of a part induced by gravitational instability (Fry 1984) and a primordial part:

$$
\begin{gathered}
B\left(\boldsymbol{k}_{1}, \boldsymbol{k}_{2}, \boldsymbol{k}_{3}\right) \simeq B_{0}\left(\boldsymbol{k}_{1}, \boldsymbol{k}_{2}, \boldsymbol{k}_{3}\right)+\left[2 J\left(\boldsymbol{k}_{1}, \boldsymbol{k}_{2}\right) P_{0}\left(k_{1}\right) P_{0}\left(k_{2}\right)+\mathrm{cyc} .\right] \\
+\int \mathrm{d}^{3} \boldsymbol{k}_{a}\left[J\left(\boldsymbol{k}_{a}, \boldsymbol{k}_{3}-\boldsymbol{k}_{a}\right) T^{\mathrm{c}}\left(\boldsymbol{k}_{a}, \boldsymbol{k}_{3}-\boldsymbol{k}_{a}, \boldsymbol{k}_{1}, \boldsymbol{k}_{2}\right)+\mathrm{cyc} .\right]
\end{gathered}
$$

where 'cyc.' denotes cyclical permutations and $J\left(\boldsymbol{k}_{a}, \boldsymbol{k}_{b}\right)$ is a function almost independent of the non-relativistic-matter density $\Omega_{0}$ and cosmological constant $\Lambda$ (Bouchet et al. 1992, 1995; Bernardeau 1994; Scoccimarro et al. 1998; Kamionkowski \& Buchalter 1998). Its detailed form need not concern us here. Here, $B_{0}$ is the primordial bispectrum (which we wish to probe) linearly evolved to redshift $z, P_{0}$ is the power spectrum, and $T^{\mathrm{c}}$ denotes the connected trispectrum, which is the Fourier transform of the connected four-point correlation function. The last two terms arise from non-linear gravitational instability (see e.g. Catelan \& Moscardini 1994). In principle, this last term $\left(\equiv B_{T}\right)$ may be important, as it grows as fast as the usual disconnected part. We will show later that this term is very small for a range of proposed models.
As already seen for the skewness, in an Einstein-de Sitter universe, $B_{0}(z) \propto(1+z)^{-3}$ and $\left[P_{0}(z)\right]^{2} \propto(1+z)^{-4}$, so the primordial bispectrum redshifts away in comparison with that arising from non-linear gravitational clustering. ${ }^{1}$

If we suppose that the galaxies (subscript g) are locally biased with respect to the mass, then the density of galaxies $\delta_{\mathrm{g}}$ can be Taylor-expanded in the mass density to second order, $\delta_{\mathrm{g}}=b_{0}+b_{1} \delta+b_{2} \delta^{2} / 2$, for some biasing coefficients $b_{i}$. The quantity $b_{0}$ affects only $\boldsymbol{k}=\boldsymbol{0}$, so it can be ignored. The bispectrum for the galaxies is then

$$
\begin{aligned}
B_{\mathrm{g}}\left(\boldsymbol{k}_{1}, \boldsymbol{k}_{2}, \boldsymbol{k}_{3}\right) \simeq & b_{1}^{3} B_{0}\left(\boldsymbol{k}_{1}, \boldsymbol{k}_{2}, \boldsymbol{k}_{3}\right) \\
& +\left\{P_{0}\left(k_{1}\right) P_{0}\left(k_{2}\right)\left[b_{1}^{3} 2 J\left(\boldsymbol{k}_{1}, \boldsymbol{k}_{2}\right)+b_{2} b_{1}^{2}\right]\right\} \\
& + \text { cyc. }+b_{1}^{3} B_{T} .
\end{aligned}
$$

So, assuming that the initial (or the linear) power spectrum is precisely known, we can do a likelihood analysis only if we also have a model for the initial (or linear) bispectrum and trispectrum as a function of the three wavenumbers. As we found previously, the primordial bispectrum redshifts away, and realistically we can detect non-Gaussianity only if $B_{T}$ is significant. In Section 4.1 we will quantify this argument further by showing that for all the models considered $B_{T} \ll B_{0}$. Let us parametrize the observed bispectrum as

$B_{\mathrm{g}}\left(\boldsymbol{k}_{1}, \boldsymbol{k}_{2}, \boldsymbol{k}_{3}\right)=P_{0}\left(k_{1}\right) P_{0}\left(k_{2}\right)\left[c_{1} 2 J\left(\boldsymbol{k}_{1}, \boldsymbol{k}_{2}\right)+c_{2}\right]+$ cyc.

In order to assess the precision with which the bias could be measured, MVH97 evaluated the precision with which the parameters $c_{1}$ and $c_{2}$ could be recovered from a likelihood analysis of the $2 \mathrm{dF}$ and/or SDSS. From their fig. 7, we conclude that if the positions of all of the galaxies in the survey volume are known, then (i) $c_{1}$ can be determined with an error of $\sim 6 \times 10^{-3}$ if $c_{2}$ is fixed; (ii) $c_{2}$ can be determined with an error of $\sim 1 \times 10^{-2}$ if $c_{1}$ is fixed; and (iii) the joint determination of the two parameters allows $c_{1}$ and $c_{2}$ to be recovered with errors of $1 \times 10^{-2}$ and $4 \times 10^{-2}$, respectively. The analysis of MVH97 further shows that the bispectrum signal comes primarily from $k \simeq 0.1-1 h \mathrm{Mpc}^{-1}$. (All of these estimates would be reduced only by a factor of about 20 if we could map the mass throughout the entire Hubble volume rather than just in the survey volume.) We will use these results, which include shot noise, to assess the smallest detectable primordial non-Gaussianity.

\subsection{CMB bispectrum}

A CMB map of the temperature $T(\hat{\boldsymbol{n}})$ as a function of position $\hat{\boldsymbol{n}}$ on the sky can be decomposed into spherical harmonics,

$\frac{\Delta T(\hat{\boldsymbol{n}})}{T}=\sum_{\ell_{m}} a_{\ell m} Y_{\ell m}(\hat{\boldsymbol{n}})$,

where the multipole coefficients are given by the inverse transformation,

$a_{\ell m}=\int \mathrm{d} \hat{\boldsymbol{n}} Y_{\ell m}^{*}(\hat{\boldsymbol{n}}) \frac{\Delta T(\hat{\boldsymbol{n}})}{T}$

${ }^{1}$ In a non-Einstein-de Sitter model, the suppression factor $(1+z)$ is replaced by $g(z)(1+z)$, but, for reasonable values of $\Omega_{0}$ and $\Lambda$, this factor is $0.3 \lesssim g(z) \lesssim 1$. Thus, we will assume an Einstein-de Sitter model from now on, noting that the general arguments are essentially unchanged in more general models. 
The CMB bispectrum $B_{\ell_{1} \ell_{2} \ell_{3}}$ is defined by

$\left\langle a_{\ell_{1}}^{m_{1}} a_{\ell_{2}}^{m_{2}} a_{\ell_{3}}^{m_{3}}\right\rangle=B_{\ell_{1} \ell_{2} \ell_{3}}\left(\begin{array}{ccc}\ell_{1} & \ell_{2} & \ell_{3} \\ m_{1} & m_{2} & m_{3}\end{array}\right)$,

where the factorization ensures statistical isotropy, and the last term is the Wigner 3J symbol. The bispectrum is an attractive statistic in that error bars can be assigned in a straightforward way, but, in the case of partial sky coverage and correlated noise, it is computationally expensive to calculate (Heavens 1998).

If we parametrize the bispectrum by an overall amplitude $f$ times some fixed function $F$, i.e. $B \ell_{1} \ell_{2} \ell_{3}=f F_{\ell_{1} \ell_{2} \ell_{3}}$, then the error on $f$ is given by (e.g. MVH97)

$$
\begin{aligned}
\sigma_{f}^{-2}= & \left\langle-\frac{\partial^{2} \ln \mathcal{L}}{\partial f^{2}}\right\rangle \simeq \sum_{\ell_{1} \leq \ell_{2} \leq \ell_{3}} \frac{\left(F_{\ell_{1} \ell_{2} \ell_{3}}\right)^{2}}{n C_{\ell_{1}} C_{\ell_{2}} C_{\ell_{3}}} \\
& \times \sum_{m_{1}, m_{2}, m_{3}} \frac{\left(\begin{array}{ccc}
\ell_{1} & \ell_{2} & \ell_{3} \\
m_{1} & m_{2} & m_{3}
\end{array}\right)^{2}}{N\left(m_{i}, \ell_{i}\right)}
\end{aligned}
$$

where $\mathcal{L}$ denotes the likelihood function and $C_{\ell} \equiv\left\langle\left|a_{\ell}^{m}\right|\right\rangle$ is the power spectrum of the sky fluctuation. Here we assume that the estimators of $B_{\ell_{1} \ell_{2} \ell_{3}}$, i.e. $a_{\ell_{1}}^{m_{1}} a_{\ell_{2}}^{m_{2}} a_{\ell_{3}}^{m_{3}}$, are uncorrelated, that the departures from Gaussianity are small and therefore that the covariance matrix can be approximated by the covariance of a Gaussian field that has the same power spectrum (Jungman et al. 1996; Heavens 1998), and we ignore the mixing that arises from partial sky coverage.

If one considers only the real part of $\left\langle a_{\ell_{1}}^{m_{1}} a_{\ell_{2}}^{m_{2}} a_{\ell_{3}}^{m_{3}}\right\rangle$ (see MVH97) then $n=1 / 2$. The quantity $N\left(m_{i}, \ell_{i}\right)$ is the number of non-zero terms like $C_{\ell_{1}} C_{\ell_{2}} C_{\ell_{3}}$ in the covariance and ranges from 1 to 30 . Equation (15) is valid as long as the noise does not dominate the signal. If $N=1$, then the sum over the Wigner $3 \mathrm{~J}$ symbols is unity; the fact that $N$ is not equal to one reduces the sum by a only few per cent, so

$\sigma_{f}^{-2} \sim 2 \sum_{\ell_{1} \leq \ell_{2} \leq \ell_{3}} \frac{\left(F_{\ell_{1} \ell_{2} \ell_{3}}\right)^{2}}{\mathrm{C}_{\ell_{1}} \mathrm{C}_{\ell_{2}} \mathrm{C}_{\ell_{3}}}$.

This expression ignores the effects of pixel noise and foreground contamination. However, the results we show below use $\ell \leqslant 100$ only, and for these modes the pixel-noise variance in $M A P$ and Planck will be negligible compared to the cosmic variance. For foregrounds, the main concern on these scales is probably dust and it is an open question whether the effects can be adequately modelled.

\section{SOME NON-GAUSSIAN MODELS}

We now proceed to consider several classes of physically motivated models with primordial non-Gaussianity in order to investigate more precisely the relative sensitivities of the CMB and LSS. Since current measurements of CMB and LSS power spectra are roughly compatible with cold dark matter (CDM) models for structure formation, we first consider CDM-like models and introduce some non-Gaussianity in several different ways. Specifically, we consider models in which the gravitational potential contains a part that is the square of a Gaussian random field, and models in which the density contains a part that is the square of a Gaussian random field. We note that such nonGaussianity may arise in slow-roll and/or non-standard (e.g. twofield) inflation models (Luo 1994; Falk, Rangarajan \& Srednicki
1993; Gangui et al. 1994; Fan \& Bardeen 1992). Moreover, both of these models may be considered as Taylor expansions of more general fields, and are thus a fairly generic form of non-Gaussianity. We also consider $O(N)-\sigma$ models, as these will approximate the non-Gaussianity expected in topological-defect models.

\subsection{Quadratic model for the potential}

We start by considering a model in which the gravitational potential $\Phi$ (in conformal Newtonian gauge) is a linear combination of a Gaussian random field $\phi$ and a term proportional to the square of the same random field,

$\Phi=\phi+\alpha_{\Phi}\left(\phi^{2}-\left\langle\phi^{2}\right\rangle\right)$,

where $\alpha_{\Phi}$ parametrizes the non-Gaussianity; in the limit $\alpha_{\Phi} \rightarrow 0$, the model becomes Gaussian. ${ }^{2}$ These models contain a primordial bispectrum for the gravitational potential:

$B_{\Phi}\left(\boldsymbol{k}_{1}, \boldsymbol{k}_{2}, \boldsymbol{k}_{3}\right) \simeq 2 \alpha_{\Phi}\left[P_{\Phi}\left(k_{1}\right) P_{\Phi}\left(k_{2}\right)+\right.$ cyc. $]$.

The leading terms in the connected trispectrum for the $\Phi$ field will be

$$
\begin{aligned}
\mathrm{T}_{\Phi}^{\mathrm{c}}\left(\boldsymbol{k}_{1}, \cdots, \boldsymbol{k}_{4}\right) \simeq & 4 \alpha_{\Phi}^{2} P_{\Phi}\left(\boldsymbol{k}_{1}\right) P_{\Phi}\left(\boldsymbol{k}_{2}\right)\left[P_{\Phi}\left(\left|\boldsymbol{k}_{1}+\boldsymbol{k}_{3}\right|\right)\right. \\
& \left.+P_{\Phi}\left(\left|\boldsymbol{k}_{1}+\boldsymbol{k}_{4}\right|\right)\right]+ \text { сус }
\end{aligned}
$$

We use a scale-invariant primordial spectrum, $P_{\Phi}=A_{H} k^{-3}$, where the amplitude is fixed by $C O B E$ to be, in our Fourier transform conventions, $A_{H} \simeq 10^{-10}$.

The relative contribution to the bispectrum of $B_{0}$ and $B_{T}$, $R \equiv B_{0} / B_{T}$, can be obtained from equations (9) and (19). By evaluating the integral we find

$R \sim\left(\alpha_{\Phi} A_{H}\right)^{-1}$.

The $B_{T}$ contribution to the bispectrum will therefore be negligible compared with $B_{0}$ as long as $\alpha_{\Phi} \ll\left(A_{H}\right)^{-1} \sim 10^{10}$.

The Fourier coefficients $\Phi(\boldsymbol{k})$ for the gravitational potential remain constant to linear order in perturbation theory in an Einsteinde Sitter universe. The Fourier coefficients $\delta(\boldsymbol{k}, z)$ of the density field evolve with time and are related to those of the gravitational potential by the Poisson equation, which can be written

$\delta(\boldsymbol{k}, z)=\mathcal{M}_{\boldsymbol{k}}(z) \Phi(\boldsymbol{k}), \quad$ where $\mathcal{M}_{\boldsymbol{k}}(z)=\frac{2 k^{2} T(k)(1+z)}{3 H_{0}^{2}}$.

In passing, we note that Poisson's equation in Fourier space ensures that the signal-to-noise ratio for measuring the nonGaussianity is the same for the density field and the potential field. The inverse of the function $\mathcal{M}_{k}(z=0)$ is plotted as a function of wavenumber $k$ in Fig. 1. Thus, the linearly evolved power spectrum for the mass, $P_{0}(k, z)$, at redshift $z$ is related to that for the gravitational potential (which remains constant in an Einsteinde Sitter universe) by

$P_{0}(k, z)=\left[\mathcal{M}_{k}(z)\right]^{2} P_{\Phi}(k)$,

where the transfer function is (e.g. Bardeen et al. 1986)

$T(k) \simeq\left(1+B k+C k^{3 / 2}+D k^{2}\right)^{-1}$,

${ }^{2}$ We note that such non-Gaussianity can arise in standard slow-roll inflation, and the parameter $\alpha_{\Phi} \sim 1$ can be related to inflaton-potential parameters (e.g. Falk et al. 1993; Gangui et al. 1994; Wang \& Kamionkowski 1999). 


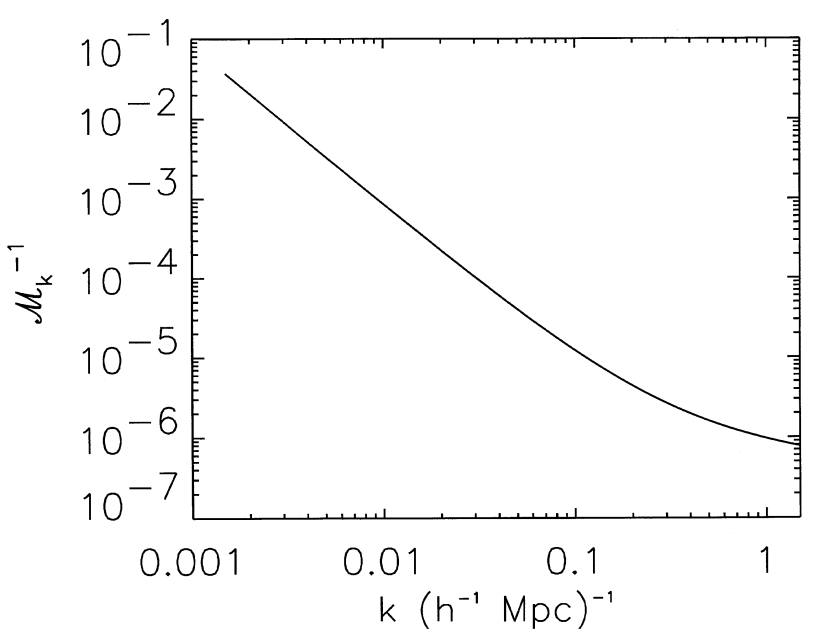

Figure 1. The inverse of the function $\mathcal{M}_{k}$ evaluated at redshift $z=0$. In the regime where second-order perturbation theory holds, this coefficient is between $10^{-5}$ and $10^{-6}$.

where $B=1.7 \mathrm{Mpc}\left(\Omega h^{2}\right)^{-1}, \quad C=9 \mathrm{Mpc}^{3 / 2}\left(\Omega h^{2}\right)^{-3 / 2}$ and $D=$ $1 \mathrm{Mpc}^{2}\left(\Omega h^{2}\right)^{-2}$. The bispectrum for the mass in this model is thus

$$
\begin{aligned}
B\left(\boldsymbol{k}_{1}, \boldsymbol{k}_{2}, \boldsymbol{k}_{3}\right) & \simeq\left\{P\left(k_{1}\right) P\left(k_{2}\right)\left[\left(2 \alpha_{\Phi} \frac{\mathcal{M}_{k_{3}}}{\mathcal{M}_{k_{1}} \mathcal{M}_{k_{2}}}\right)+2 J\left(\boldsymbol{k}_{1}, \boldsymbol{k}_{2}\right)\right]\right\} \\
& + \text { cyc. }
\end{aligned}
$$

Comparing equation (24) with equation (1), we see that this particular form of primordial non-Gaussianity leads to a presentday bispectrum that looks like a scale-dependent non-linear bias. In particular, for equilateral configurations, equation (24) becomes identical to equation (11) if we identify $c_{2}=2 \alpha_{\Phi} / \mathcal{M}_{k}$. We can therefore use the results of MVH97 to determine the smallest $\alpha_{\Phi}$ that would be detectable by $2 \mathrm{dF} / \mathrm{SDSS}$, under the assumption that there is no non-linear biasing. ${ }^{3}$ Because of phase-space considerations, most of the signal comes from the largest wavenumbers for which second-order perturbation theory holds, i.e. $k \sim 0.6$ or $\mathcal{M}_{k}^{-1} \simeq 10^{-5}-10^{-6}$. Thus, we conclude that the smallest $\alpha_{\Phi}$ that would give rise to an observable signal in the 2dF/SDSS bispectrum is $\alpha_{\Phi} \sim 10^{3}-10^{4}$.

The primordial gravitational-potential bispectrum in equation (18) will lead to a non-zero bispectrum in the CMB via the SachsWolfe effect, and this bispectrum can be calculated to be (Luo 1994; Wang \& Kamionkowski 1999), ${ }^{4}$

$$
\begin{aligned}
B_{\ell_{1} \ell_{2} \ell_{3}}= & \sqrt{\frac{\left(2 \ell_{1}+1\right)\left(2 \ell_{2}+1\right)\left(2 \ell_{3}+1\right)}{4 \pi}}\left(\begin{array}{ccc}
\ell_{1} & \ell_{2} & \ell_{3} \\
0 & 0 & 0
\end{array}\right) \\
& \times \frac{2 \alpha_{\Phi}}{A_{\mathrm{SW}}}\left[C_{\ell_{1}} C_{\ell_{2}}+C_{\ell_{1}} C_{\ell_{3}}+C_{\ell_{2}} C_{\ell_{3}}\right],
\end{aligned}
$$

where $A_{\mathrm{SW}} \simeq 1 / 3$ is the Sach-Wolfe coefficient. Plugging this

\footnotetext{
${ }^{3}$ In the analysis of MVH97, equilateral and degenerate (a repeated vector and one of twice the amplitude and opposite direction) configurations are used; since the inverse of the function $\mathcal{M}_{k}$ is not strongly $k$-dependent on the scales where most of the signal comes from, $c_{2}=2 \alpha_{\Phi} / \mathcal{M}_{k}$ is still a good approximation.

${ }^{4}$ Actually, our calculation ignores the physics that gives rise to the acoustic peaks at $l \gtrsim 100$. However, we restrict our analysis to $l \lesssim 100$. A more accurate treatment would change our results by no more than an order of magnitude, and this level of precision is sufficient for our purposes.
}

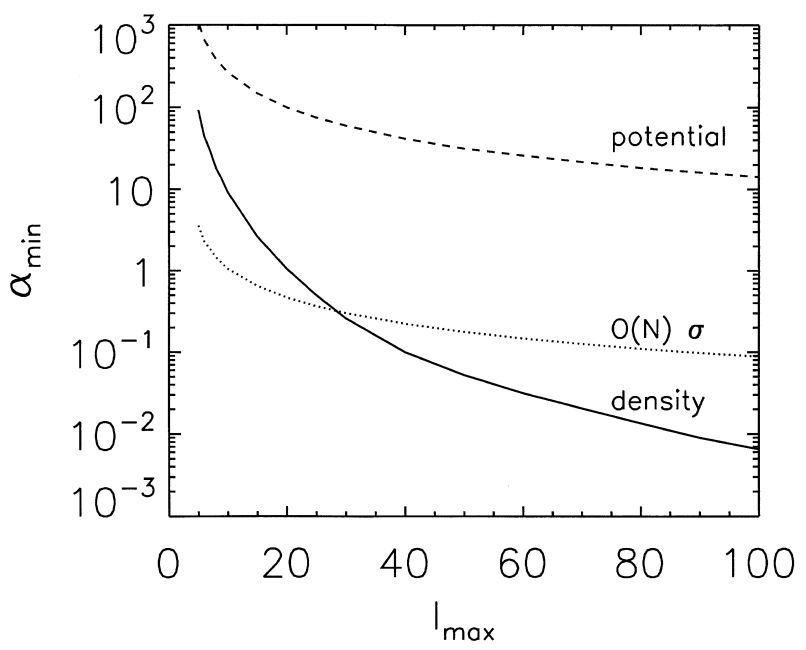

Figure 2. The smallest non-Gaussian amplitude $\alpha$ that can be detected with the $\mathrm{CMB}$ as a function of the largest multipole moment $\ell$ used in the analysis. The solid line is for a model in which the present-day fractional overdensity field contains a term that is the square of a Gaussian random field; the dashed line is for a model in which the gravitational potential contains a term that is the square of Gaussian random field; and the dotted line refers to the $O(N) \sigma$ models.

bispectrum (and a scale-invariant set of $C_{\ell}$ ) into equation (16), we learn that the smallest $\alpha_{\Phi}$ that could be detectable with a CMB map (using only $\ell \leq 100$ ) is $\sim 20 .^{5}$ (The dashed curve in Fig. 2 shows the smallest $\alpha_{\Phi}$ detectable with the CMB as a function of the largest multipole moment $\ell$ used in the analysis.) Thus, we conclude that the CMB will be at least two orders of magnitude more sensitive to a non-zero value of $\alpha_{\Phi}$ than LSS.

\subsection{Quadratic model for the density}

We now consider an alternative model in which the density field (rather than the gravitational potential) contains a term that is the square of a Gaussian random field:

$\delta=\phi+\alpha_{\delta}\left(\phi^{2}-\left\langle\phi^{2}\right\rangle\right)$,

where now $\phi$ is some other Gaussian random field. Such a model has been considered in some two-field inflation models (e.g. Luo \& Schramm 1993). ${ }^{6}$

Since the density perturbation evolves with time in linear theory (unlike the gravitational potential), we must specify the epoch at which the density perturbation $\delta$ is related to $\phi$ through equation (26). We choose this epoch to be the current epoch, $z=0$. Doing so, the spatial mass bispectrum today due to primordial nonGaussianity is

$B_{0}\left(\boldsymbol{k}_{1}, \boldsymbol{k}_{2}, \boldsymbol{k}_{3}\right) \simeq 2 \alpha_{\delta} P\left(k_{1}\right) P\left(k_{2}\right)+$ cyc.

and Luo \& Schramm (1993) have shown that the contribution to the present-day bispectrum from the primordial trispectrum is negligible compared with this $\left(B_{T} \ll B_{0}\right)$, as long as 2OPT holds.

\footnotetext{
${ }^{5}$ This result is in rough agreement with the conclusion that the $\alpha \sim$ 10-100 (Luo 1994; Gangui et al. 1994; Mollerach et al. 1995) nonGaussian signal from non-linear evolution prior to $z \simeq 1100$ is at best marginally detectable (Spergel \& Goldberg 1999a,b).

${ }^{6}$ Here $\alpha_{\delta}$ is a phenomenological parameter that varies from model to model; for example, it could be arbitrarily large in some multifield inflation models (e.g. Peebles 1998a,b).
} 
By comparing equation (27) with equation (11), we see that this form of primordial non-Gaussianity gives rises to a present-day skewness that mimics precisely that due to a scale-independent non-linear bias. Again, from the results of MVH97, the smallest detectable $\alpha_{\delta}$ in this model is $\sim 0.01$.

Now let us consider the CMB bispectrum of this model. The spatial bispectrum for the gravitational potential here is

$B_{\Phi}\left(\boldsymbol{k}_{1}, \boldsymbol{k}_{2}, \boldsymbol{k}_{3}\right)=\frac{2 \alpha_{\delta} \mathcal{M}_{k_{1}} \mathcal{M}_{k_{2}}}{\mathcal{M}_{k_{3}}}\left[P_{\Phi}\left(k_{1}\right) P_{\Phi}\left(k_{2}\right)\right]+$ cyc.

Although we have not carried out an exact calculation, it is easily seen (at least in the $\ell \gg 1$ limit) that

$$
\begin{aligned}
B_{\ell_{1} \ell_{2} \ell_{3}} \simeq & \sqrt{\frac{\left(2 \ell_{1}+1\right)\left(2 \ell_{2}+1\right)\left(2 \ell_{3}+1\right)}{4 \pi}}\left(\begin{array}{ccc}
\ell_{1} & \ell_{2} & \ell_{3} \\
0 & 0 & 0
\end{array}\right) \\
& \times \frac{2 \alpha_{\delta}}{A_{\mathrm{SW}}}\left[\frac{2}{3} C_{\ell_{1}} C_{\ell_{2}} \frac{\ell_{1}^{2} \ell_{2}^{2}}{\ell_{3}^{2}}+\text { cyc. }\right]
\end{aligned}
$$

Applying equation (16), we find that the smallest detectable $\alpha_{\delta}$ in this model is $\sim 0.01$ (using only $\ell \leq 100$; results for other $\ell_{\max }$ are shown in Fig. 2), which is comparable to the LSS error. However, since this model has more power in the CMB bispectrum at larger $\ell$, the smallest detectable $\alpha_{\delta}$ decreases by roughly an order of magnitude even if we go out only to $\ell_{\max }=200$. Also, in this case we find that the CMB will provide a more precise probe of a primordial bispectrum. Moreover, as a corollary, this particular result demonstrates that if the maps provided by MAP and Planck are consistent with Gaussian, then any measurement of a non-zero $c_{2}$ from the LSS bispectrum can be interpreted unambiguously as evidence for non-linear biasing, rather than as some primordial non-Gaussianity.

\section{3 $O(N)-\sigma$ models}

The $O(N)-\sigma$ model provides an approximation to the nonGaussianity expected in topological-defect or scalar-field-alignment models (Turok \& Spergel 1991; Jaffe 1994). The $N=1$ model has domain walls; $N=2$ has global strings; $N=3$ has global monopoles; $N=4$ has global textures; and higher $N$ correspond to non-topological-defect models. For large $N$ this model approaches the Gaussian model, so we take $\alpha_{\sigma}=N^{-1 / 2}$, and as $\alpha_{\sigma} \rightarrow 0$ the models become asymptotically Gaussian. Since calculations of power spectra and higher-order statistics for the CMB and LSS are quite involved for these models, our analysis will be only approximate. As we will see below, these order-ofmagnitude estimates will be sufficiently precise for our purposes.

For equilateral-triangle configurations and for values of $k$ that can be probed with $2 \mathrm{dF} / \mathrm{SDSS}$, an ansatz for the LSS power spectrum, bispectrum and trispectrum in the linear regime is (Jaffe 1994)

$P \simeq 12.5 \mathcal{K}^{2} k T^{2}(k), \quad B_{0} \simeq 1.6 \mathcal{K}^{3} \alpha_{\sigma} T^{3}(k)$,

$T^{\mathrm{c}} \simeq \mathcal{K}^{4} \frac{1}{k \alpha_{\sigma}^{2}} T^{4}(k)$,

where $\mathcal{K} \simeq 30-100 \mathrm{Mpc}^{2} h^{-2}$ [although Jaffe (1994) did not include the transfer functions]. From equations (30) and (31), $B_{T}$ is found to be always $\leqq 0.4 B_{0}$. Since $B_{0}$ will be the dominant contribution to the bispectrum we conclude that the non-Gaussianity of this model will act like a scale-dependent non-linear-bias contribution with

$c_{2} \simeq B_{0} / P^{2} \simeq \alpha_{\sigma}\left[\mathcal{K} k^{2} 10^{2} T(k)\right]^{-1}$.

For the scales probed by SDSS/2dF, $c_{2} \lesssim \alpha_{\sigma} / 300$, and therefore the minimum $\alpha_{\sigma}$ detectable from LSS will be $\sim 30$.

Precise calculation of the CMB bispectrum for these models is well beyond the scope of this paper. To obtain an order-ofmagnitude estimate, we assume that the spatial polyspectra for this model, equations (30)-(31), give rise to potential polyspectra through the Poisson equation, and then that the CMB anisotropy is proportional to the potential perturbation at the surface of last scatter. Doing so, we find ${ }^{7}$

$$
\begin{aligned}
B_{\ell_{1} \ell_{2} \ell_{3}} \sim & \sqrt{\frac{\left(2 \ell_{1}+1\right)\left(2 \ell_{2}+1\right)\left(2 \ell_{3}+1\right)}{4 \pi}}\left(\begin{array}{ccc}
\ell_{1} & \ell_{2} & \ell_{3} \\
0 & 0 & 0
\end{array}\right) \\
& \times 5000 \alpha_{\sigma}\left[C_{\ell_{1}} C_{\ell_{2}} C_{\ell_{3}}\right]^{2 / 3} .
\end{aligned}
$$

Using equation (16), we find that the smallest detectable $\alpha_{\sigma}$ would be $\sim 0.1$ (using multipole moments up to $\ell_{\max }=100$; the dependence on $\ell_{\max }$ is indicated in Fig. 2). Thus, we again conclude that the $\mathrm{CMB}$ will provide a more precise probe of a primordial non-Gaussianity.

\section{CONCLUSIONS}

We addressed the question of which of the CMB and LSS is better poised to detect primordial non-Gaussianity of several varieties. We used the bispectrum as a discriminating statistic since it is the lowest-order quantity that has zero expectation value for a Gaussian field. We considered three forms of non-Gaussianity: one in which the gravitational potential contained a term that was the square of a Gaussian field; another in which the density field was the square of a Gaussian field; and a third that resembled that expected from topological defects. We showed that, in all cases, the CMB is likely to provide a better probe of such nonGaussianity. One of these models produced a mass bispectrum that mimicked a scale-dependent non-linear bias, and the others mimicked a scale-independent non-linear bias.

Of course, our results are not fully general. In principle, it is possible to think of some other type of non-Gaussianity for which our conclusions would not hold, such as, for example, a model in which the primordial fluctuations are Gaussian only on CMB scales. However, plausible physical mechanisms that produce nearly scale-invariant power spectra should generically produce non-Gaussian signals that have scale dependences roughly like those that we investigated. Thus we may conclude that if CMB maps turn out to be consistent with Gaussian initial conditions, any non-Gaussianity seen in the LSS bispectrum can be unambiguously attributed to the effects of biasing.

\section{ACKNOWLEDGMENTS}

We would like to thank A. Jaffe, A. Kosowsky and R. Scoccimarro for useful comments. LV acknowledges the support of a TMR grant. LV and AFH thank the Physics Department of Columbia University for hospitality. This work was supported at Columbia

${ }^{7}$ Details will be presented elsewhere. Our estimate differs from that of Luo (1994) for reasons that escape us. We have checked, however, that our conclusions are unaltered if we use his results. 
by a DoE Outstanding Junior Investigator Award, DE-FG0292ER40699, NASA grant NAG5-3091, and the Alfred P. Sloan Foundation.

\section{REFERENCES}

Albrecht A., Stebbins A., 1992, Phys. Rev. Lett., 68, 2121

Allen T. J., Grinstein B., Wise M. B., 1987, Phys. Lett. B, 197, 66

Bardeen J. M., Steinhardt P. J., Turner M. S., 1983, Phys. Rev. D, 28, 679

Bardeen J. M., Bond J. R., Kaiser N., Szalay A. S., 1986, ApJ, 304, 15

Bernardeau F., 1994, ApJ, 433, 1

Bouchet F., Juszkiewicz R., Colombi S., Pellat R., 1992, ApJ, 394, L5

Bouchet F., Colombi S., Hivon S. C. E., Juszkiewicz R., 1995, A\&A, 296, 575

Bromley B., Tegmark M., 1999, ApJ, 524, L79

Catelan P., Moscardini L., 1994, ApJ, 426, 14

Colombi F., Szapudi I., Szalay A., 1998, MNRAS, 296, 253

Falk T., Rangarajan R., Srednicki M., 1993, ApJ, 403, L1

Fan Z., Bardeen J. M., 1992, University of Washington preprint

Ferreira P., Magueijo J., Gorski K., 1998, ApJ, 503, L1

Fry J. N., 1984, ApJ, 279, 499

Fry J. N., Scherrer R. J., 1994, ApJ, 429, 36

Gangui A., Lucchin F., Matarrese S., Mollerach S., 1994, ApJ, 430, 447

Gaztanaga E., Fosalba P., 1998, MNRAS, 301, 524

Goroff M. H., Grinstein B., Rey S. J., Wise B., 1986, ApJ, 311, 6

Guth A., Pi S. Y., 1982, Phys. Rev. Lett., 464, L11

Hawking S. W., 1982, Phys. Rev. Lett., 115B, 295

Heavens A. F., 1998, MNRAS, 299, 805
Hill C. T., Schramm D. N., Fry J. N., 1989, Comments Nucl. Part. Phys., 19,25

Jaffe A. H., 1994, Phys. Rev. D, 49, 3902

Jungman G., Kamionkowski M., Kosowsky A., Spergel D. N., 1996, Phys. Rev. D, 54, 1332

Kamionkowski M., Buchalter A., 1999, ApJ, 514, 7

Kamionkowski M., Jaffe A. H., 1998, Nat, 395, 639

Kofman L., Pogosyan D., 1988, Phys. Lett. B, 214, 508

Luo X., 1994, ApJ, 427, L71

Luo X., Schramm D. N., 1993, ApJ, 408, 33

Matarrese S., Verde L., Heavens A., 1997, MNRAS, 290, 651 (MVH97)

Mollerach S., Gangui A., Lucchin F., Matarrese S., 1995, ApJ, 453, 1

Pando J., Valls-Gabaud D., Fang L. Z., 1998, Phys. Rev. Lett., 81, 4568

Peebles P. J. E., 1980, The Large Scale Structure of the Universe. Princeton Univ. Press, Princeton

Peebles P. J. E., 1998a, ApJ, 510, 523

Peebles P. J. E., 1998b, ApJ, 510, 531

Salopek D., Bond J. R., Bardeen J. M., 1989, Phys. Rev. D, 40, 1753

Scoccimarro R., Colombi S., Fry J. N., Frieman J. A., Hivon E., Melott A., 1998, ApJ, 496, 586

Spergel D. N., Goldberg D. M., 1999a, Phys. Rev. D, 59, 103001

Spergel D. N., Goldberg D. M., 1999b, Phys. Rev. D, 59, 103002

Starobinsky A. A., 1982, Phys. Lett. B, 117, 175

Turok N., 1989, Phys. Rev. Lett., 63, 2625

Turok N., Spergel D. N., 1991, Phys. Rev. Lett., 66, 3093

Vachaspati T., 1986, Phys. Rev. Lett., 57, 1655

Vilenkin A., 1985, Phys. Rep., 121, 263

Wang L., Kamionkowski M., 1999, astro-ph/9907431

This paper has been typeset from a $\mathrm{T}_{\mathrm{E}} \mathrm{X} / \mathrm{L} \mathrm{T} \mathrm{EX}$ file prepared by the author. 